\title{
Balanced Nanocomposite Thermosetting Materials for HVDC and AC Applications
}

\author{
G. C Stevens*, N. A. Freebody \\ Gnosys Global Ltd \\ Guildford, UK \\ g.stevens@gnosysgroup.com
A. S. Vaughan, S. Virtanen
University of Southampton
Southampton, UK

\author{
A. Hyde, F. Perrot, I. Szkoda-Giannaki \\ Alstom Grid Research and Technology Centre \\ Stafford, UK
}

\author{
P. Baker \\ Mekufa UK Ltd \\ Gloucester, UK
}

\author{
S. A. F. Bon, S. R. Coles, J. A. Medlam \\ University of Warwick \\ Coventry, UK
}

There is a need to develop materials with controlled electrical resistivity, reduced space charge accumulation, higher thermal conductivity, higher dielectric strength and enhanced voltage endurance to cope with DC stresses in High Voltage Direct Current (HVDC) transmission systems in addition to HVAC requirements. If the balance of properties, performance and process requirements are achieved this may lead to HVDC insulation systems and equipment having a reduced footprint, larger power densities, and greater multi-stress resilience with longer service lifetimes. It reports findings of a project that is engaging this challenge and investigates the development and scaling of new thermoset based nanocomposite electrical insulation materials for HVDC power transmission applications. Some of the results such as increased electrical breakdown strength and reduced electrical conductivity for reactively surface functionalised nanosilica, and increased thermal conductivity for nano boron nitride and their significance in regard to the wider application of these electrical insulation materials are also discussed. With sufficient understanding of these properties, their trade-offs and process requirements it is possible to tailor balanced materials for specific use in HVAC or HVDC components.

Keywords - Nanocomposite, epoxy resin, HVDC, HVAC, power transmission, Nanodielectric

\section{INTRODUCTION}

Nanodielectrics are multi-component materials containing nanostructures which influence their dielectric, electrical and other physical properties. Epoxy-based composites are widely used as medium and high voltage insulators for HVDC and HVAC network applications. Conventional materials are based upon micro-composites filled with micron-sized particulates of materials such as alumina and silica have been extensively studied; the drivers being to modify the electrical, thermal and mechanical properties of the neat epoxy resins and to reduce cost [1]. However, while the inclusion of micron-sized inorganic particulates leads to improvements in thermal and mechanical characteristics, it can have a negative impact on key electrical parameters such as electrical breakdown strength and space charge accumulation for HVDC applications. Similarly, conventional epoxy resins may not have the purity for HVDC applications and improved resins may be required.

Nanodielectrics based on epoxy resin systems can readily be prepared by dispersing nano- and meso-sized inorganic fillers in the polymer matrix. A key feature of nanodielectrics, is the large specific surface area of nanoscale fillers and the many properties and performance factors linked to interfacial effects. This includes the characteristics of the so called interphase regions between the nanofiller surface and the bulk matrix [2]. In this region it is likely that some properties may adopt values that are not equivalent to bulk matrix. When the interfacial area is large, the proportion of interphase in the system will be commensurately large such that, in principle, the material is likely to exhibit unique combinations of properties. Hence the technological interest in such systems.

A significant body of published work also recognizes the potential benefits of the nanophase in increasing resistance to surface corona and partial discharge [3], enhanced voltage endurance [4] and the potential mitigation of space charge formation [5] in addition to the potential tailoring of other properties such as permittivity, dielectric loss, thermal conductivity, dielectric strength, mechanical properties and thermal stability. This will be illustrated alongside the need for balanced properties in regard to functionalized reactive and unreactive nano-fillers how to transferring this to engineering practice. 
However, a consequence of the above is that the nanoparticles must be well dispersed if such property benefits are to be realized. Part of this challenge relates to understanding several quantitative structure-property-process relationships for these materials and our ability to manipulate them controllably in complex nanodielectrics. So, controlled dispersion of the nanophase and its qualification is central to this as is the understanding of nanophase surface chemistry and the interphase region between the nanofiller and the polymer matrix. Appropriate surface chemistry to enhance interfacial coupling to the matrix and its control is also essential. There is also a need for reliable materials processing and industrial scaling of the process methods used.

\section{NANOCOMPEIM PROJECT}

\section{A. Background and Objectives}

The NanocompEIM project is a Technology Strategy Board (TSB) Energy Materials research project which addresses the need to achieve systematic processing and production of nanocomposite electrical insulation materials to ensure the reliable production of next generation HVDC equipment having enhancements in performance properties to enhance power equipment ratings.

The aim of the project is to translate current understanding and practical experience of the processing of nanocomposite electrical insulation materials in order to develop a set of materials design and process rules to achieve reliable production and process scaling in component manufacture. Scalable processing methods have been developed to produce components using materials with controlled dispersion and interfacial characteristics to achieve appropriate property and performance enhancements.

Achieving this will be of immense benefit to power equipment manufacturers and network operators that use this equipment, particularly in the context of renewable generation integration and the move to develop Smart Grid and Low Carbon Networks.

\section{B. Materials}

For simplicity the work reported here is focused primarily on the Huntsman CY1300 bisphenol A diglycidyl ether epoxy resin with the corresponding Huntsman Aradur A-917 anhydride hardener and 1-Methylidiazole catalyst (available from Sigma Aldrich). In uncured samples nanofillers were dispersed into the resin via a planetary mixer at $3600 \mathrm{rpm}$ for 3 minutes prior to analysis. For cured samples the nanofillers were dispersed into the resin as with the uncured samples. Following this the hardener and catalyst were added, mixed via shear mixing at $4000 \mathrm{rpm}$ for 20 minutes, degassed, cured at 80 degrees for 2 hours and post cured at 120 degrees $\mathrm{C}$. Cured samples were then cut to size and prepared in accordance with the methods appropriate to the analysis method.

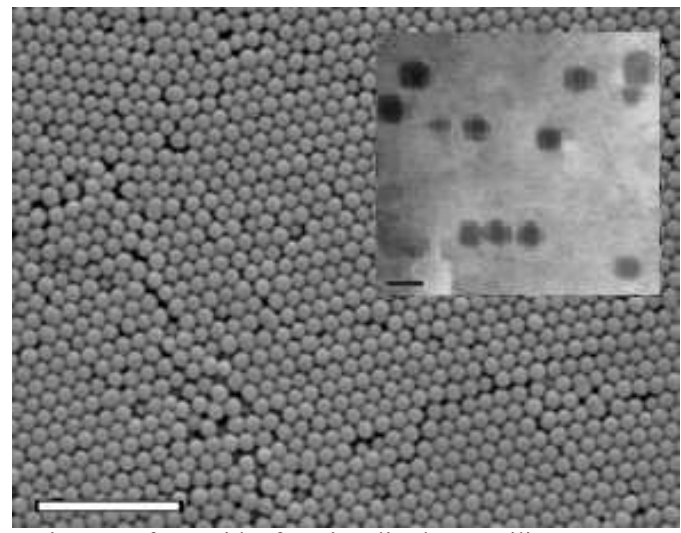

Fig. 1. SEM image of epoxide functionalised nanosilica evaporated onto silica substrate, scale bar equivolent to 1 micron. Inset in top right corner is a comparison with TEM (scale bar $=100 \mathrm{~nm}$ ).

As with the mechanical methods of nanoparticle dispersion, chemical dispersion and functionalization methods for nanoparticles has also been investigated with nanosilica and compared against the untreated filler. Although many different methods of the chemical treatment of nanofillers were investigated, we report on two methods which show clear benefit for use in HVDC and HVAC nanocomposites. The first method involves the use of (3glycidyloxypropyl)trimethoxysilane (GLYMO) and the second is an epoxy functionalized, silane treated nanosilica (EFST). Glymo treatment was applied to a powdered nanosilica (supplied by NanoAmor with an average particle size of 80 $\mathrm{nm}$ ) whereas the silane treatment of nanosilicas was achieved in the liquid phase and was provided commercially. These materials were also compared against a commercially available nanopox nanosilica filler in dispersed in resin. An example scanning electron microscopy (SEM) image of one of the epoxy functionalised silane treated nanosilicas and a transmission electron microscopy (TEM) image of the fillerepoxy composite can be seen in Figure 1. The particles are well defined and stable with little or no aggregation occurring which has previously been identified as a potential cause for reduction in physical properties. Untreated boron nitride supplied by Momentive was also investigated.

\section{Dispersion}

Several methods of dispersion, both mechanical and chemical, have been trialled throughout this project. Mechanical methods include sonication, high shear mixing, planetary mixing, and a combination of all 3 mechanical methods. Sonication works by using ultrasonic pulses to rapidly compress and expand local regions of the sample resulting in micro bubble cavitation in the liquid medium providing high local shear and a driving force for homogenizing the system. High shear mixing however, uses a high speed rotor to mix the fluid in question. The velocity of the fluid at the outside of the rotor will be higher than the velocity of the fluid at the centre of the rotor. It is this difference in fluid velocity which creates a shear force to disperse any fillers within the resin matrix. Finally, the use of planetary mixing appears to be the most effective method trialled. During planetary mixing high speed rotation combined with the counter revolution generates a large 
centrifugal force of up to $400 \mathrm{G}$ and establishes a vertical spiral convection mixing pattern that rapidly disperses the components uniformly. In the case of nanoparticle dispersion, during this mixing the particles collide with each under high local shear force, assisting the breaking up of aggregates and agglomerates. It is important to ensure that the best dispersion of the nanofiller is achieved as any agglomeration/aggregation of the nanofillers can lead to a decrease in the various final properties of the material.

All samples were incorporated into the CY1300 resin and the solvent from both chemical methods was extracted by rotary evaporation before the materials were mixed via planetary mixing. They were then cast and cured into thin films for electrical and thermal testing. Chemical functionalization helps to increase the interface between the host resin matrix and the nanoparticles. Current literature indicates that the more closely bound the nanoparticles are to the host polymer matrix, the more stable it will be and the better the improvement in physical properties will be [2]. In this work scaling from typically $0.1 \mathrm{~kg}$ to tens of kilograms batch size was demonstrated.

\section{Material testing}

In HVDC applications and to a large extend in HVAC applications, there are pressing needs, for dielectric materials having:

- $\quad$ enhanced thermal conductivity

- $\quad$ reduced electrical conductivity

- enhanced breakdown strength

- tailored bulk and surface resistivity's

- $\quad$ space-charge mitigation

- improved voltage endurance

- resistance to surface discharges

A wide variety of materials containing various nanofiller types, loadings and surface treatments were investigated and subjected to a variety of measurements including, thermal, electrical, mechanical and a variety of spectral and optical methods to check the dispersion of the materials. It is important to analyze any potential new nanocomposites for use in HVDC applications using these measurements so that its performance in components can be properly predicted. It is very important to obtain a balanced outcome of property as significant increases in some properties may lead to decreases in others.

Out of all the materials tested it was found that nanosilica treated with either the GLYMO or the epoxide functionalized silane gave the best performance in comparison with the unfilled resin and untreated fillers, with respect to the electrical properties with no significant decrease in thermal conductivity or mechanical properties whereas the Nanopox yielded a decrease in electrical properties. Some of the enhancements of electrical breakdown strength and DC resistivity can be seen in Figures 2 and 3 as examples of the

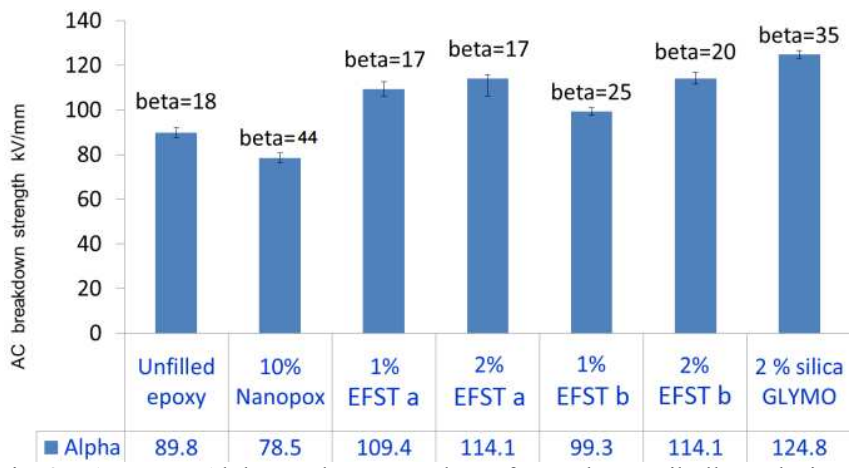

Fig. 2. Average Alpha and Beta values from the Weibull analysis of electrical breakdown data of resin filled with treated nanosilicas.

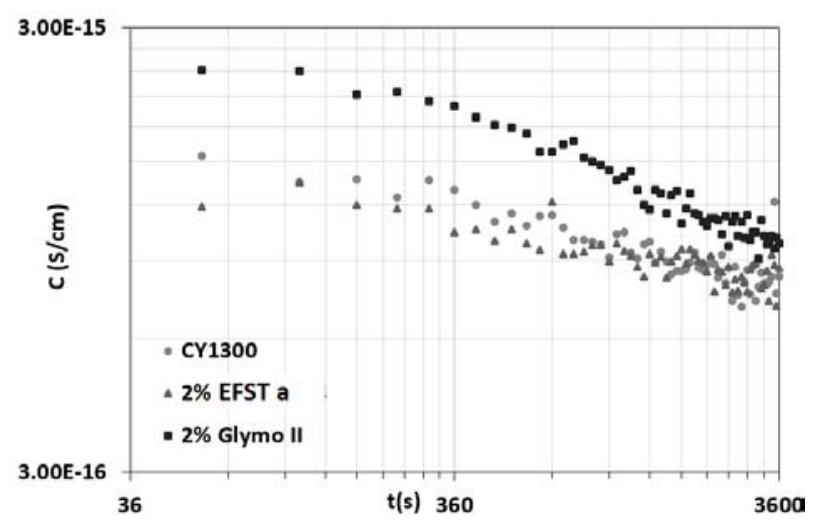

Fig. 3. DC Conductivity plot of resin filled with treated nanosilicas.

enhancements of electrical breakdown strength and DC resistivity can be seen in Figures 2 and 3 as examples of the improvement of electrical properties. Electrical breakdown strength is also a useful metric when considering materials for use in HVAC applications and can help inform the failure probability of components in service, whereas measurements such as space charge accumulation and DC conductivity are more important in the consideration of HVDC components. For all electrical measurements it was found that with both silica and boron nitride (BN), the improvements in electrical properties rises with filler loading up until approximately $2 \%$ by weight when electrical properties seem to decrease again, most likely due to agglomeration.

With regards to thermal measurements, samples containing $\mathrm{BN}$ had a notably higher thermal conductivity. This increase in thermal conductivity without a significant drop in electrical breakdown strength makes BN a strong contender for use in the electrical power applications, work in this area is still in its infancy and there are several research groups currently working to utilize $\mathrm{BN}$ and maximize its effectiveness in this area. It is beyond the scope of this paper to provide an in depth explanation for the various changes in properties and will be commented on fully in a full paper currently in production.

The advantage of performing all of these measurements is that a large body of knowledge can be built enabling us to better tailor the material to its specific use. By fully 
understanding property trade-offs for the materials in question it will be possible to combine nanofillers and treatment methods such that balanced properties can be achieved and the material can be optimized for the application requirements.

\section{NANO-MICRO COMPOSITE MATERIALS}

In NanocompEIM a number of options for the deployment of the nanomaterials in future applications for HVDC and HVAC have been identified. It is important to stress that although the focus of NanocompEIM is on HVDC applications, both the range of nanomaterials and the nature of the applications will also have a positive impact on traditional HVAC applications. Some examples of future potential deployments include:

- HVDC Valve components (Saturable reactors)

- $\quad$ GIS Equipment (Barriers) for AC \& DC applications

- Substation composite (post) insulators

Demonstration nanocomposite components for comparative testing with micro composites were selected according to the type of performance being required e.g. heat conduction, electrical breakdown or surface corona resistance.

Typically these components contain micro fillers to ensure the coefficient of thermal expansion is matched with that of metal inserts. In industry a silica micro filler is usually used for this purpose. It is important therefore to ensure that the addition of nanofillers into these systems does not adversely affect the properties of the micro fillers. It is also necessary to show that any processes used are scalable from the laboratory for potential future use in industry. This project has achieved this by the generation of master batches several kilograms in size which were then mixed and cured into demonstrator components at Mekufa, examples of which can be seen in Figure 4. Current work on the project involves the complete testing of these demonstrator pieces and their comparison with demonstrators made with the industry standard such that the use of nanocomposites can be assessed.

\section{CONCLUSIONS}

Nanoparticle surface coupling or encapsulation will assist master batch stability. Modification of the interface between polymer and filler will also help to ensure good nanodispersion. Although many surface treatments assist dispersion, their use may not result in any improvement in electrical breakdown strength. In comparison, the use of GLYMO was able to achieve stable dispersions and an improvement in breakdown strength as was the use of epoxide functionalized nanosilicas.

Processing with commercial planetary centrifugal mixers have been shown to be an effective method for nanoparticle dispersion and extending shelf life. It is a fast dispersion



Fig. 4. Example demonstrator components cast from masterbatch nano-micro composite material formulations.

method that can be easily scaled to the $20 \mathrm{~kg}$ batch scale, which would suit fabrication of industrial scale quantities of master batch. The process naturally degasses the nanoparticle mixture and it is also possible to process in a vacuum. The method is well suited to highly viscous systems, i.e. naturally suiting epoxy resins and processing of systems with high solids content.

All materials have been subjected to a wide array of physical property measurements. Properties such as permittivity, dielectric loss, thermal conductivity, dielectric strength, mechanical properties and thermal stability have all been investigated. Whilst all of these properties are necessary the imperative is the important balance between these properties, essential for a successful material. With sufficient understanding of these properties, their trade-offs and process requirements it is possible to tailor material for specific use in HVAC or HVDC components.

\section{ACKNOWLEDGMENT}

The authors thank G. Tzemis (National Grid), D. Walker (Scottish Power Power Systems), A. Robertson (Scottish \& Southern Energy Power Distribution), and Innovate UK (formerly the Technology Strategy Board), for their continued support and funding for the NanocompEIM project.

\section{REFERENCES}

[1] T. Rouyre, A. C. Taylor, M. Fu, F. Perrot, I. James, "Nano-and microsilica modification of epoxy polymers," IEEE International Conference on Solid Dielectrics, pp.1-4, 2010

[2] C. Yeung, "Spectroscopic analysis of nanodielectric interfaces." Doctoral Thesis, University of Southampton, pp. 223. 2013

[3] M. F. Frechette, R. Y. Larocque, M. Blouin, S. Boily, "Nanophase semi-conductive ceramics: dielectric surface performance when exposed to charges," Annual Report Conference on Electrical Insulation and Dielectric Phenomena, pp.326-331 Oct 2004.

[4] J. K. Nelson and Y. Hu, "Nanocomposite dielectrics - properties and implications" J. Phys. D: Appl. Phys. Vol. 38 pp. 21, 2005

[5] D. Fabiani, G. C. Montanari, A. Dardano, G. Guastavino, L. Testa, and M. Sangermano, "Space Charge Dynamics in Nanostructured Epoxy Resin," Annual Report Conference on Electrical Insulation and Dielectric Phenomena, pp.710-713, Oct 2008. 